\title{
Full dynamic reactions in the main links of big band saw machines
}

\author{
Boycho Marinov* \\ Institute of Mechanics, Bulgarian Academy of Sciences.
}

Global Journal of Engineering and Technology Advances, 2021, 09(03), 092-104

Publication history: Received on 08 November 2021; revised on 18 December 2021; accepted on 20 December 2021

Article DOI: https://doi.org/10.30574/gjeta.2021.9.3.0162

\begin{abstract}
In this study, the full dynamic reactions in the bearing supports of the leading wheel of big band saw machines are determined. These reactions are caused by both the external loads and the kinematic and mass characteristics of the rotating disk. Expressions for calculating the dynamic reactions caused by external forces and moments that occur in the operating mode are obtained. The influence of the kinematic and mass characteristics of the rotating disk is studied and expressions for calculating the dynamic reactions caused by these characteristics are obtained. Expressions for calculating the full dynamic reactions that load the bearing supports of the leading wheel are obtained. With the help of these expressions, the parameters of the band saw machine can be selected in such a way as to ensure a minimum load on the shaft and bearings.
\end{abstract}

Keywords: Band saw machines; External load; Kinematics and mass characteristics; Dynamic reactions

\section{Introduction}

The band saw machines are a certain class of woodworking machines for longitudinal, cross or curvilinear sawing. They are used for sawing logs, boards, slabs, prisms, details etc. These machines saw the workpiece through a band saw blade and two leading wheels. According to the diameters of the two wheels and the width of the band saw blade, band saw machines are divided into three groups:

- $\quad$ Ordinary band saw machines, $400 \leq D \leq 1000[\mathrm{~mm}], B$ is up to 40 [mm];

- Deal band saw machines, $1000 \leq D \leq 1500[\mathrm{~mm}], 70 \leq B \leq 175[\mathrm{~mm}]$;

- Log band saw machines or big band saw machines, $1100 \leq D \leq 3500[\mathrm{~mm}], 140 \leq B \leq 360[\mathrm{~mm}]$.

Research related to the determination of the external loads that form the dynamic reactions in main links of some classes of woodworking machines are published in the technical literature. Zheng at al. [1] propose an acceptance method of adjusting the dynamic tensile force range of band saw blades. This method is to calculate the static and dynamic tensile force of band saw blades by the string vibration formula. With a focus on industrial applications, Marchal at al. [2] emphasize the wood peeling process, which is a very demanding special case of wood cutting. Porankiewicz at al. [3] evaluate non-linear dependencies between main (tangential) and normal (radial) cutting forces up on two machining parameters by up-routing and down-routing wood of Douglas fir (Pseudotsuga menziesii) and Oak (Quercus petraea). A new computational model, based on fracture mechanics, is used by Hlásková at al. [4] to determine cutting forces. The new model uses two main parameters: fracture toughness and shear yield stresses. Moradpour at al. [5] measure the components of cutting force in bandsaw processing of green oak and beech wood at $90^{\circ}-90^{\circ}$ cutting direction. Results show that all cutting force components change by increasing the cutting speed and feed rate over the analysed range. Krenke at al. [6] present a method for the characterization of test setups, enabling a correction method of different

\footnotetext{
* Corresponding author: Boycho Marinov

Institute of Mechanics, Bulgarian Academy of Sciences.
} 
examinations. Subsequently, the identified transmission characteristic is used for the correction of own cutting force measurements.

The purpose of this study is to propose analytical and numerical solution to analyze the full dynamic reactions in the bearing supports of the upper leading wheel. In order to achieve this purpose, the following main tasks must be solved:

- $\quad$ obtaining expressions for calculating the dynamic reactions due to the external load;

- $\quad$ obtaining expressions for calculating the dynamic reactions due to the kinematic and mass characteristics of the rotating disk;

- $\quad$ obtaining expressions for calculating the full dynamic reactions in the bearing supports;

- $\quad$ construction of plane and spatial diagrams, which show the change of the full dynamic reactions when changing different parameters.

The received expressions for calculating the full dynamic reactions can be used both when designing new machines and to ensure the efficient and reliable work of the band saw machines in operating mode.

\section{Expose}

\subsection{Scheme of the cutting mechanism}

The scheme of the cutting mechanism is shown in Fig.1[7, 8]. The following symbols are defined: 1-main shaft, 2 and 5belt pulleys, 3 and 4-leading wheels, $A$ - band saw blade, and 6-upper shaft.

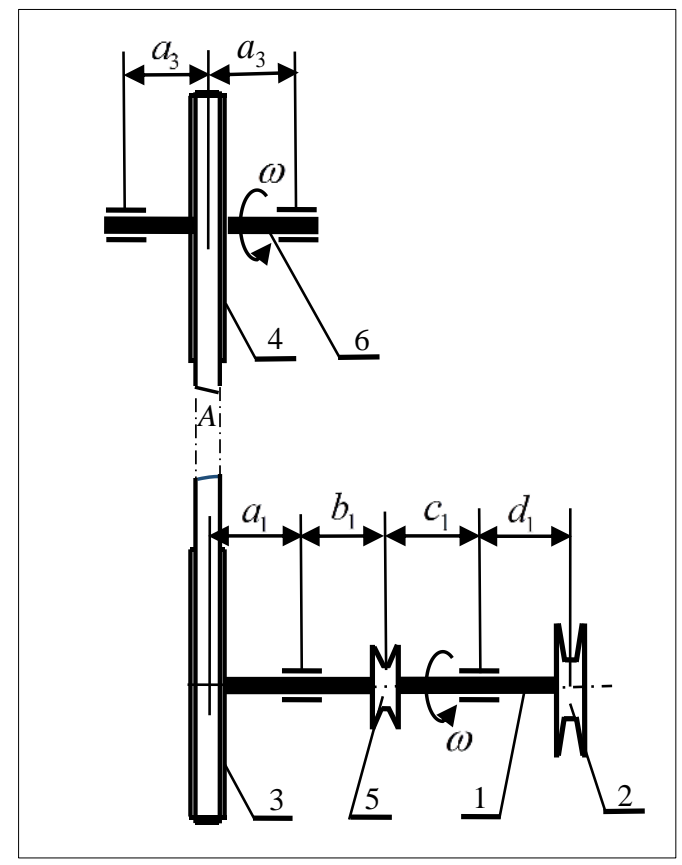

Figure 1 Cutting mechanism

\subsection{Dynamic model}

Figure 2 shows the dynamic model when the upper leading wheel is mounted symmetrically between the two bearing supports. 


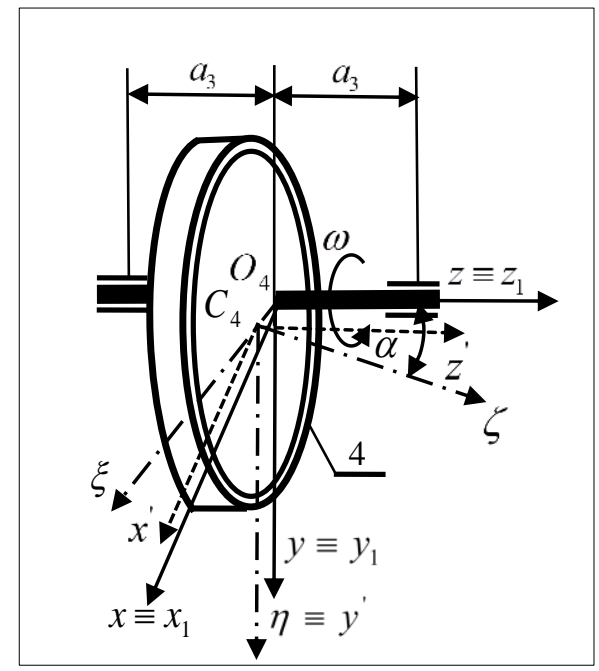

Figure 2 Dynamic model

The following coordinate systems are used to obtain expressions for calculating the full dynamic reactions [9]:

- $O_{4} x y z$ - fixed coordinate system;

- $O_{4} x_{1} y_{1} z_{1}$ - moving coordinate system;

- $\quad C_{4} x^{\prime} y^{\prime} z^{\prime}$ - coordinate system, whose axes are parallel to the axes of the moving coordinate system;

- $C_{4} \xi \eta \varsigma$ - coordinate system, whose axes are principal axes of inertia.

The linear deviation (eccentricity) $e$ and angular deviation $\alpha$ are also shown in the figures as $e=O_{4} C_{4}$ and $\alpha$ is the angle between the axes $\zeta$ and $z$.

\subsection{Dynamic reactions caused by external load}

We consider the external forces that load the leading wheel. These forces are shown in Fig. 3

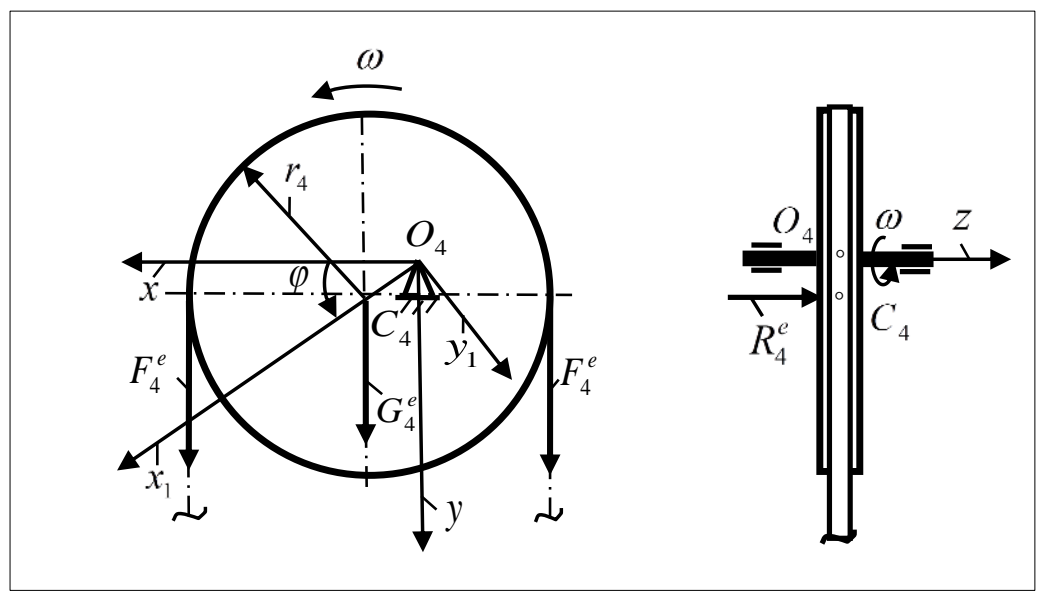

Figure 3 External load on the leading wheel

The leading wheel rotates with a constant angular velocity $\omega$ and describes an angle $\varphi=\omega t$. The tensile forces in the band saw blade are denoted by $F_{4}^{e}$. Difference expressions for calculating this force are presented in the technical literature. An appropriate formula for its calculation is written below [7]. 


$$
F_{4}^{e}=1.5 N_{c} / V,
$$

where $N_{c}$ is the cutting power and $V$ is the cutting speed.

The weight of the leading wheel $G_{4}^{e}$ can be calculated from a formula known in literature, $G_{4}^{e}=m_{4} g[10,11]$, where $m_{4}$ is the mass of the wheel and $g$ is the acceleration of gravity.

The force $R_{4}^{e}$ is transmitted from band saw blade to the leading wheel. This force can be calculated with sufficient accuracy from the following dependence [7].

$$
R_{4}^{e}=\frac{R_{b}^{n}+R_{\Sigma}}{2}
$$

where $R_{\Sigma}$ is the total resistance force. This force is calculated for each individual case. $R_{b}^{n}$ is the normal force loading the workpiece. This force can be calculated from the expression below, in which the cutting force $P_{b}^{\tau}$ participates. [7, $12]$.

$$
R_{b}^{n}=m P_{b}^{\tau}=m \frac{K_{\Delta(\lambda)} b H u}{V},
$$

where $H$ is the thickness of the workpiece, $u$ is the feeding speed, $b$ is the width of the cutter. $\boldsymbol{m}$ is a coefficient that changes within the following limits $0 \leq m \leq 1 . K_{\Delta(\lambda)}$ is the specific work of the cutting. It is determined from the expressions below [13].

$$
K_{\Delta}=k+\frac{a_{\rho} p}{u_{z \Delta}}+\frac{\alpha_{\Delta} H}{b}, \quad K_{\lambda}=k+\frac{a_{\rho} p s}{b u_{z \lambda}}+\frac{\alpha_{\lambda} H}{b} .
$$

The symbol $\alpha_{\Delta(\lambda)}$ is the friction intensity of the shavings on the sawing walls, as $\alpha_{\Delta}$ is used for swage-set teeth and $\alpha_{\lambda}$ - for part-set teeth. $a_{\rho}$ is a coefficient of the blunt teeth, $\boldsymbol{S}$ is a thickness of the band saw blades. $k$ is the fictitious pressure on the front side of the teeth and $p$ is the fictitious specific force on the back side of the teeth. The feeding of one tooth is marked with $u_{z \Delta}, u_{z \lambda}$.

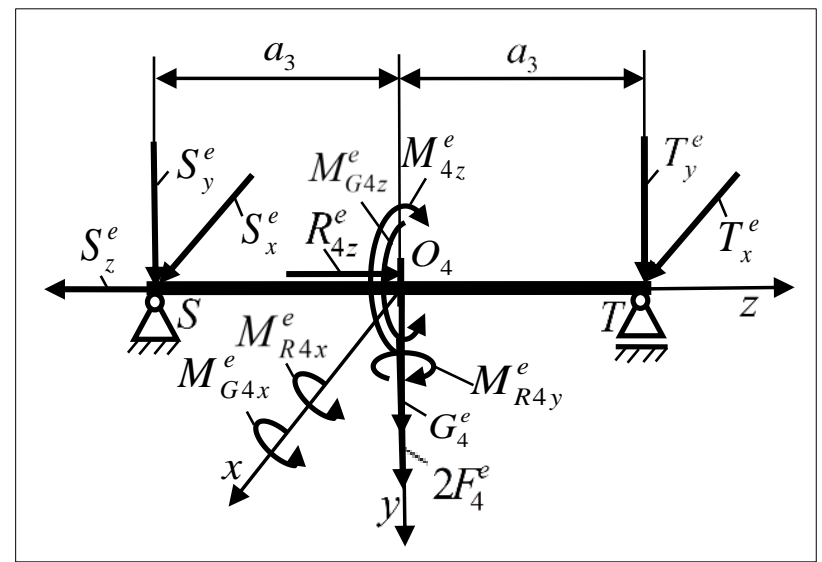

Figure 4 Loading the upper shaft

To determine the dynamic reactions, we first determine the load on the upper shaft of the band saw machine. For this purpose, we reduce the forces that load the leading wheel to the axis of rotation of the upper shaft. Figure 4 shows the 
forces and moments that load the shaft and cause the dynamic reactions in the bearing supports. These reactions are also shown.

The force $R_{4 z}^{e}$ is calculated from the following expression:

$$
R_{4 z}^{e}=R_{4}^{e} \cos \alpha
$$

This force creates the following moments relative to the axes $x, y$ and $z$.

$$
\begin{aligned}
& M_{R 4 x}^{e}=\frac{1}{2}\left(\frac{m K_{\Delta(\lambda)} b H u}{V}+R_{\Sigma}\right) e \cos \alpha \sin \omega t, \\
& M_{R 4 y}^{e}=\frac{1}{2}\left(\frac{m K_{\Delta(\lambda)} b H u}{V}+R_{\Sigma}\right) \cos \alpha\left(r_{4}+e \cos \omega t\right), \\
& M_{R 4 z}^{e}=0 .
\end{aligned}
$$

The weight of the leading wheel $G_{4}^{e}$ creates moments relative to the axes $x, y$ and $z$ presented below.

$$
\begin{aligned}
& M_{G 4 x}^{e}=m_{4} g e \sin \alpha, \\
& M_{G 4 y}^{e}=0, \\
& M_{G 4 z}^{e}=m_{4} g e \cos \alpha \cos \omega t .
\end{aligned}
$$

Dynamic reactions can be determined from the equilibrium conditions. From these conditions, we obtain the corresponding equations in which the support reactions participate.

$$
\begin{aligned}
& R_{4 z}^{e}-S_{z}^{e}=0, \\
& M_{G 4 x}^{e}+M_{R 4 x}^{e}-G_{4}^{e} a_{3}-2 F_{4}^{e} a_{3}-T_{y}^{e} 2 a_{3}=0, \\
& M_{G 4 x}^{e}+M_{R 4 x}^{e}+G_{4}^{e} a_{3}+2 F_{4}^{e} a_{3}+S_{y}^{e} 2 a_{3}=0, \\
& -M_{R 4 y}^{e}+T_{x}^{e} 2 a_{3}=0, \\
& -M_{R 4 y}^{e}-S_{x}^{e} 2 a_{3}=0 .
\end{aligned}
$$

After performing some substitutions, we get the expressions for the dynamic reactions caused by the external load in a final form. These expressions are presented below.

$$
\begin{gathered}
S_{z}^{e}=\left(\frac{m K_{\Delta(\lambda)} b H u}{V}+R_{\Sigma}\right) \frac{\cos \alpha}{2}, \\
T_{y}^{e}=\frac{m_{4} g}{2 a_{3}}\left(e \sin \alpha-a_{3}\right)-\frac{1.5 N_{c}}{V}+\frac{1}{4 a_{3}}\left(\frac{m K_{\Delta(\lambda)} b H u}{V}+R_{\Sigma}\right) e \cos \alpha \sin \omega t, \\
S_{y}^{e}=-\frac{m_{4} g}{2 a_{3}}\left(e \sin \alpha+a_{3}\right)-\frac{1.5 N_{c}}{V}-\frac{1}{4 a_{3}}\left(\frac{m K_{\Delta(\lambda)} b H u}{V}+R_{\Sigma}\right) e \cos \alpha \sin \omega t, \\
T_{x}^{e}=\frac{r_{4}}{4 a_{3}}\left(\frac{m K_{\Delta(\lambda)} b H u}{V}+R_{\Sigma}\right) \cos \alpha+\frac{1}{4 a_{3}}\left(\frac{m K_{\Delta(\lambda)} b H u}{V}+R_{\Sigma}\right) e \cos \alpha \cos \omega t,
\end{gathered}
$$




$$
S_{x}^{e}=-\frac{r_{4}}{4 a_{3}}\left(\frac{m K_{\Delta(\lambda)} b H u}{V}+R_{\Sigma}\right) \cos \alpha-\frac{1}{4 a_{3}}\left(\frac{m K_{\Delta(\lambda)} b H u}{V}+R_{\Sigma}\right) e \cos \alpha \cos \omega t .
$$

\subsection{Dynamic reactions caused by the kinematic and mass characteristics of the rotating disk}

To determine the dynamic reactions caused by the kinematic and mass characteristics of the leading wheel, we use the dynamic model, shown in Fig.5.

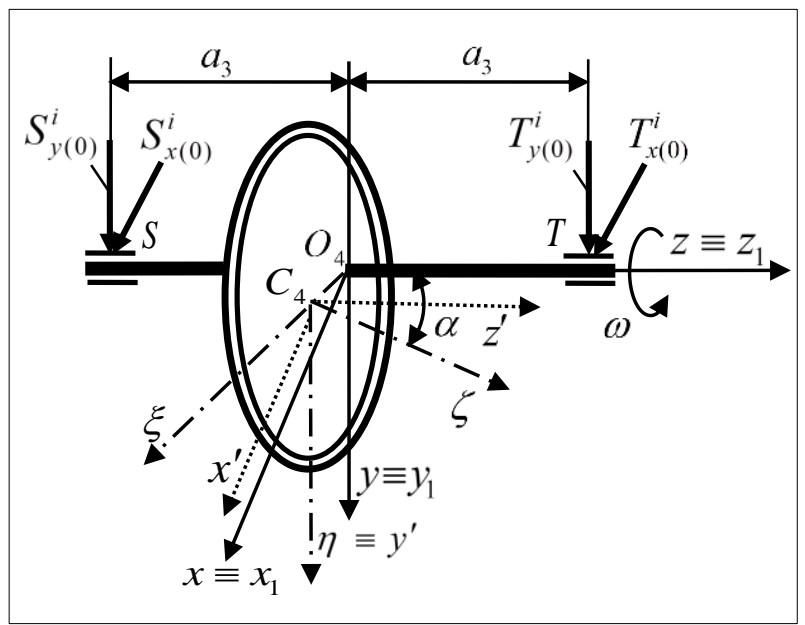

Figure 5 Dynamic model

Dynamic reactions can be determined by the following equations [10]:

$$
\begin{aligned}
& m_{4}\left(-\varepsilon y_{C_{4}}^{(0)}-\omega^{2} x_{C_{4}}^{(0)}\right)=S_{x(0)}^{i}+T_{x(0)}^{i}, \\
& m_{4}\left(\varepsilon x_{C_{4}}^{(0)}-\omega^{2} y_{C_{4}}^{(0)}\right)=S_{y(0)}^{i}+T_{y(0)}^{i}, \\
& -\varepsilon J_{x z}^{(0)}+\omega^{2} J_{y z}^{(0)}=S_{y(0)}^{i} a_{3}-T_{y(0)}^{i} a_{3}, \\
& -\varepsilon J_{y z}^{(0)}-\omega^{2} J_{x z}^{(0)}=-S_{x(0)}^{i} a_{3}+T_{x(0)}^{i} a_{3} .
\end{aligned}
$$

The following symbols are used: $x_{C_{4}}^{(0)}$ and $y_{C_{4}}^{(0)}$ are the coordinates of the centre of mass $C_{4}$ in the initial moment. The centrifugal moments of the leading wheel about axes $x, z$ and $y, z$ are denoted by $J_{x z}^{(0)}$ and $J_{y z}^{(0)}$ in the initial moment.

We consider motion with constant angular velocity i.e., $\omega=$ const, $\varepsilon=0$ where $\varepsilon$ is the angular acceleration. In this case, the dynamic reactions are constant with respect to the moving coordinate system. These reactions change about the fixed coordinate system depending on the change in the angle $\varphi$. We can determine their sizes to an arbitrary position. Therefore, we choose the initial position when $\varphi=0$. For this position, the axes of the fixed coordinate system $O_{4} x y z$ and the moving coordinate system $O_{4} x_{1} y_{1} z_{1}$ coincide. In this case, the coordinates of the centre of mass $C_{4}$ are calculated by the following expressions:

$$
x_{C_{4}}^{(0)}=e \cos \alpha, y_{C_{4}}^{(0)}=0, z_{C_{4}}^{(0)}=-e \sin \alpha .
$$

The centrifugal moments $J_{x z}^{(0)}$ and $J_{y z}^{(0)}$ for the initial position $(\varphi=0)$ can be calculated using the Huygens-Steiner theorem $[10,11]$.

$$
J_{x z}^{(0)}=J_{x^{\prime} z^{\prime}}^{(0)}+m_{4} x_{C_{4}}^{(0)} z_{C_{4}}^{(0)}, \quad J_{y z}^{(0)}=J_{y^{\prime} z^{\prime}}^{(0)}+m_{4} y_{C_{4}}^{(0)} z_{C_{4}}^{(0)}
$$


where $J_{x z}^{(0)}$ and $J_{y z^{\prime}}^{(0)}$ are the centrifugal moments relative to the respective axes of the coordinate system $C_{4} x^{\prime} y^{\prime} z^{\prime}$. These moments are calculated from the following dependencies:

$$
J_{x^{\prime} z^{\prime}}^{(0)}=\int_{(V)} x^{\prime} z^{\prime} d m, \quad J_{y^{\prime} z^{\prime}}^{(0)}=\int_{(V)} y^{\prime} z^{\prime} d m .
$$

We need to express the coordinates $x^{\prime}, z^{\prime}$ and $y^{\prime}$ through the coordinates $\xi, \zeta$ and $\eta$. The two coordinate systems $C_{4} \xi \zeta \eta$ and $C_{4} x^{\prime} y^{\prime} z^{\prime}$ have a common origin and are rotated relative to one other at an angle $\alpha$, which is shown in Fig. 6.

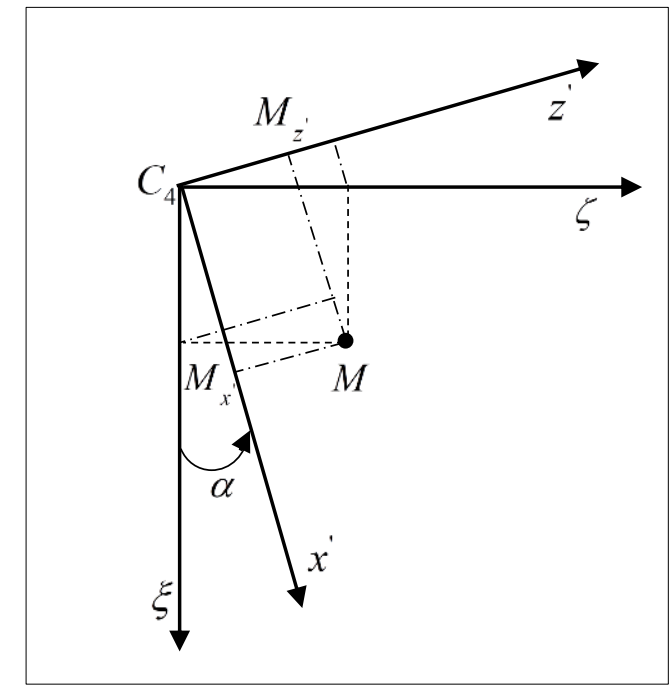

Figure 6 Transition of the coordinates between the two rectangular coordinate systems

The transition from the first rectangular coordinates $x^{\prime}, z^{\prime}, y^{\prime}$ to the other rectangular coordinates $\xi$, $\zeta, \eta$ takes place according to known formulas, which are written below.

$$
\begin{aligned}
& x^{\prime}=\xi \cos \alpha+\zeta \sin \alpha, \\
& z^{\prime}=-\xi \sin \alpha+\zeta \cos \alpha, \\
& y^{\prime}=\eta .
\end{aligned}
$$

We replace these expressions in expressions (13) and obtain the following dependences for calculating the centrifugal moments of inertia $J_{x z^{\prime}}^{(0)}$ and $J_{y^{\prime}}^{(0)}$.

$$
\begin{aligned}
& J_{x^{\prime} z^{\prime}}^{(0)}=\int_{(V)}(\xi \cos \alpha+\zeta \sin \alpha)(-\xi \sin \alpha+\zeta \cos \alpha) d m, \\
& J_{y^{\prime} z^{\prime}}^{(0)}=\int_{(V)} \eta(-\xi \sin \alpha+\zeta \cos \alpha) d m .
\end{aligned}
$$

We transform the above expressions and obtain the expressions for the centrifugal moments, keeping in mind the fact that the axes $\xi, \eta$ and $\zeta$ are the principal axes of inertia.

$$
J_{x^{\prime} z^{\prime}}^{(0)}=\frac{1}{2}\left(J_{\xi}-J_{\zeta}\right) \sin 2 \alpha, \quad J_{y^{\prime} z^{\prime}}^{(0)}=0 .
$$

We substitute the above expressions in the expressions (12) and receive the dependencies for calculating the centrifugal moments $J_{x z}^{(0)}$ and $J_{y z}^{(0)}$. 


$$
J_{x z}^{(0)}=\frac{1}{2}\left(J_{\xi}-J_{\zeta}-m_{4} e^{2}\right) \sin 2 \alpha, \quad J_{y z}^{(0)}=0,
$$

where $J_{\xi}$ and $J_{\zeta}$ are the mass moments of inertia of the rotating disk toward axes $\xi$ and $\zeta$.

After the explanations made above, we can write the expressions (10) in the following form.

$$
\begin{aligned}
& -m_{4} \omega^{2} e \cos \alpha=S_{x(0)}^{i}+T_{x(0)}^{i}, \\
& 0=S_{y(0)}^{i}+T_{y(0)}^{i}, \\
& 0=S_{y(0)}^{i} a_{3}-T_{y(0)}^{i} a_{3}, \\
& -\frac{\omega^{2}}{2}\left(J_{\xi}-J_{\zeta}-m_{4} e^{2}\right) \sin 2 \alpha=-S_{x(0)}^{i} a_{3}+T_{x(0)}^{i} a_{3} .
\end{aligned}
$$

From these expressions, we obtain the expressions for the dynamic reactions for the initial position $(\varphi=0)$ in the final form.

$$
\begin{aligned}
& S_{x(0)}^{i}=\frac{\omega^{2}}{2}\left[\frac{1}{a_{3}}\left(J_{\xi}-J_{\zeta}-m_{4} e^{2}\right) \sin \alpha-m_{4} e\right] \cos \alpha, \\
& T_{x(0)}^{i}=-\frac{\omega^{2}}{2}\left[\frac{1}{a_{3}}\left(J_{\xi}-J_{\zeta}-m_{4} e^{2}\right) \sin \alpha+m_{4} e\right] \cos \alpha, \\
& S_{y(0)}^{i}=T_{y(0)}^{i}=0 .
\end{aligned}
$$

The dynamic reactions for an arbitrary position $(\varphi \neq 0)$ can be determined from the following dependencies.

$$
\begin{aligned}
& S_{x}^{i}=S_{x(0)}^{i} \cos \omega t, S_{y}^{i}=S_{x(0)}^{i} \sin \omega t, \\
& T_{x}^{i}=T_{x(0)}^{i} \cos \omega t, T_{y}^{i}=T_{x(0)}^{i} \sin \omega t .
\end{aligned}
$$

We can write the expressions for the dynamic reactions in final form using the dependencies (19) and (20).

$$
\begin{aligned}
& S_{x}^{i}=\frac{\omega^{2}}{2}\left[\frac{1}{a_{3}}\left(J_{\xi}-J_{\zeta}-m_{4} e^{2}\right) \sin \alpha-m_{4} e\right] \cos \alpha \cos \omega t, \\
& S_{y}^{i}=\frac{\omega^{2}}{2}\left[\frac{1}{a_{3}}\left(J_{\xi}-J_{\zeta}-m_{4} e^{2}\right) \sin \alpha-m_{4} e\right] \cos \alpha \sin \omega t, \\
& T_{x}^{i}=-\frac{\omega^{2}}{2}\left[\frac{1}{a_{3}}\left(J_{\xi}-J_{\zeta}-m_{4} e^{2}\right) \sin \alpha+m_{4} e\right] \cos \alpha \cos \omega t, \\
& T_{y}^{i}=-\frac{\omega^{2}}{2}\left[\frac{1}{a_{3}}\left(J_{\xi}-J_{\zeta}-m_{4} e^{2}\right) \sin \alpha+m_{4} e\right] \cos \alpha \sin \omega t .
\end{aligned}
$$

\subsection{Full dynamic reactions}

The full dynamic reactions are sum by the dynamic reactions caused by the external load and dynamic reactions caused by the kinematic and mass characteristics of the rotating disk. They can be determined by the following dependencies. 


$$
\begin{aligned}
& S_{x}=-\frac{r_{4}}{4 a_{3}}\left(\frac{m K_{\Delta(\lambda)} b H u}{V}+R_{\Sigma}\right) \cos \alpha-\left\{\frac{1}{4 a_{3}}\left(\frac{m K_{\Delta(\lambda)} b H u}{V}+R_{\Sigma}\right) e \cos \alpha-\right. \\
& \left.-\frac{\omega^{2}}{2}\left[\frac{1}{a_{3}}\left(J_{\xi}-J_{\zeta}-m_{4} e^{2}\right) \sin \alpha-m_{4} e\right] \cos \alpha\right\} \cos \omega t, \\
& S_{y}=-\frac{m_{4} g}{2 a_{3}}\left(e \sin \alpha+a_{3}\right)-\frac{1.5 N_{c}}{V}-\left\{\frac{1}{4 a_{3}}\left(\frac{m K_{\Delta(\lambda)} b H u}{V}+R_{\Sigma}\right) e \cos \alpha-\right. \\
& \left.-\frac{\omega^{2}}{2}\left[\frac{1}{a_{3}}\left(J_{\xi}-J_{\zeta}-m_{4} e^{2}\right) \sin \alpha-m_{4} e\right] \cos \alpha\right\} \sin \omega t, \\
& T_{x}=\frac{r_{4}}{4 a_{3}}\left(\frac{m K_{\Delta(\lambda)} b H u}{V}+R_{\Sigma}\right) \cos \alpha+\left\{\frac{1}{4 a_{3}}\left(\frac{m K_{\Delta(\lambda)} b H u}{V}+R_{\Sigma}\right) e \cos \alpha-\right. \\
& \left.-\frac{\omega^{2}}{2}\left[\frac{1}{a_{3}}\left(J_{\xi}-J_{\zeta}-m_{4} e^{2}\right) \sin \alpha+m_{4} e\right] \cos \alpha\right\} \cos \omega t, \\
& T_{y}=\frac{m_{4} g}{2 a_{3}}\left(e \sin \alpha-a_{3}\right)-\frac{1.5 N_{c}}{V}+\left\{\frac{1}{4 a_{3}}\left(\frac{m K_{\Delta(\lambda)} b H u}{V}+R_{\Sigma}\right) e \cos \alpha-\right. \\
& \left.-\frac{\omega^{2}}{2}\left[\frac{1}{a_{3}}\left(J_{\xi}-J_{\zeta}-m_{4} e^{2}\right) \sin \alpha+m_{4} e\right] \cos \alpha\right\} \sin \omega t \text {. }
\end{aligned}
$$

\section{Numerical solutions}

The results of the carried out computer experiments are presented in Figs.7 and 8. We use the following initial data [1316]:

$\omega=56\left[\mathrm{~s}^{-1}\right], e=\{0,0.001\}[\mathrm{m}], \alpha=\{0,0.017\}[\mathrm{rad}], a_{3}=0.6[\mathrm{~m}], m_{4}=810[\mathrm{~kg}], J_{\xi}=171\left[\mathrm{kgm}^{2}\right], J_{\varsigma}=336\left[\mathrm{kgm}^{2}\right]$, $N_{c}=43,4[\mathrm{~kW}], V=45[\mathrm{~m} / \mathrm{s}], \quad r_{4}=0.8[\mathrm{~m}], \quad s=1.47[\mathrm{~mm}], \quad b=2.5[\mathrm{~mm}], \quad p=9220[\mathrm{~N} / \mathrm{m}]$, $k=35 \times 10^{6}[\mathrm{~Pa}] ， \quad \alpha_{\lambda}=245 \times 10^{3}[\mathrm{~Pa}], \quad \alpha_{\Delta}=196 \times 10^{3}[\mathrm{~Pa}], m=0.5, \quad H=0.42[\mathrm{~m}], \quad u=0.5[\mathrm{~m} / \mathrm{s}]$ ， $u_{z \Delta}=2.5[\mathrm{~mm}], K_{\Delta}=80 \times 10^{6}\left[\mathrm{~J} / \mathrm{m}^{3}\right], R_{\Sigma}=2400[\mathrm{~N}]$.

\subsection{Plane diagrams}

Figure 7 shows the plane diagrams of the full dynamic reactions $S_{x}, S_{y}, T_{x}, T_{y}$ 

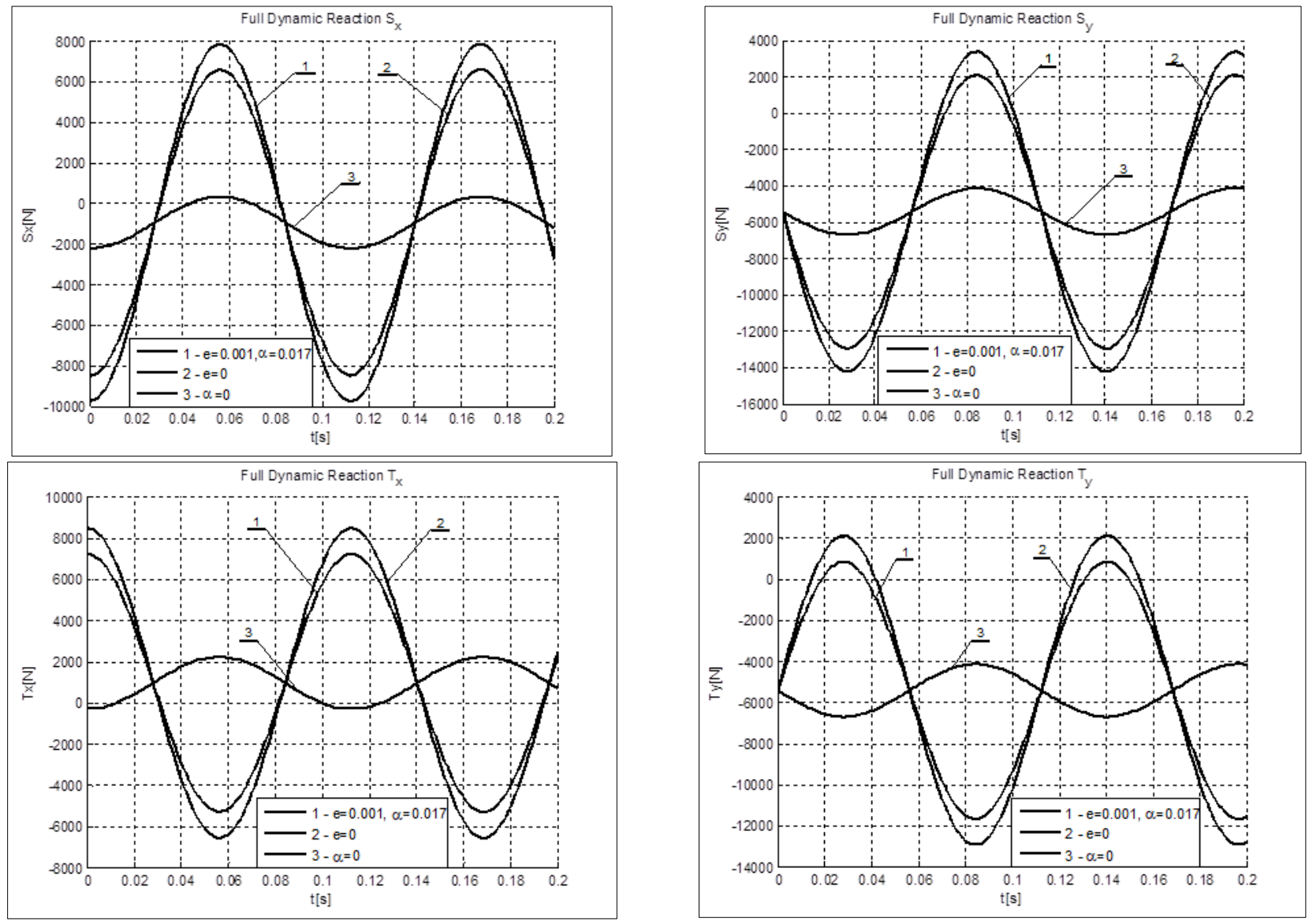

Figure 7 Full dynamic reactions $S_{x}, S_{y}, T_{x}, T_{y}$

\subsection{Spatial diagrams}

Figure 8 shows the spatial diagrams of the full dynamic reactions $S_{x}, S_{y}, T_{x}, T_{y}$.
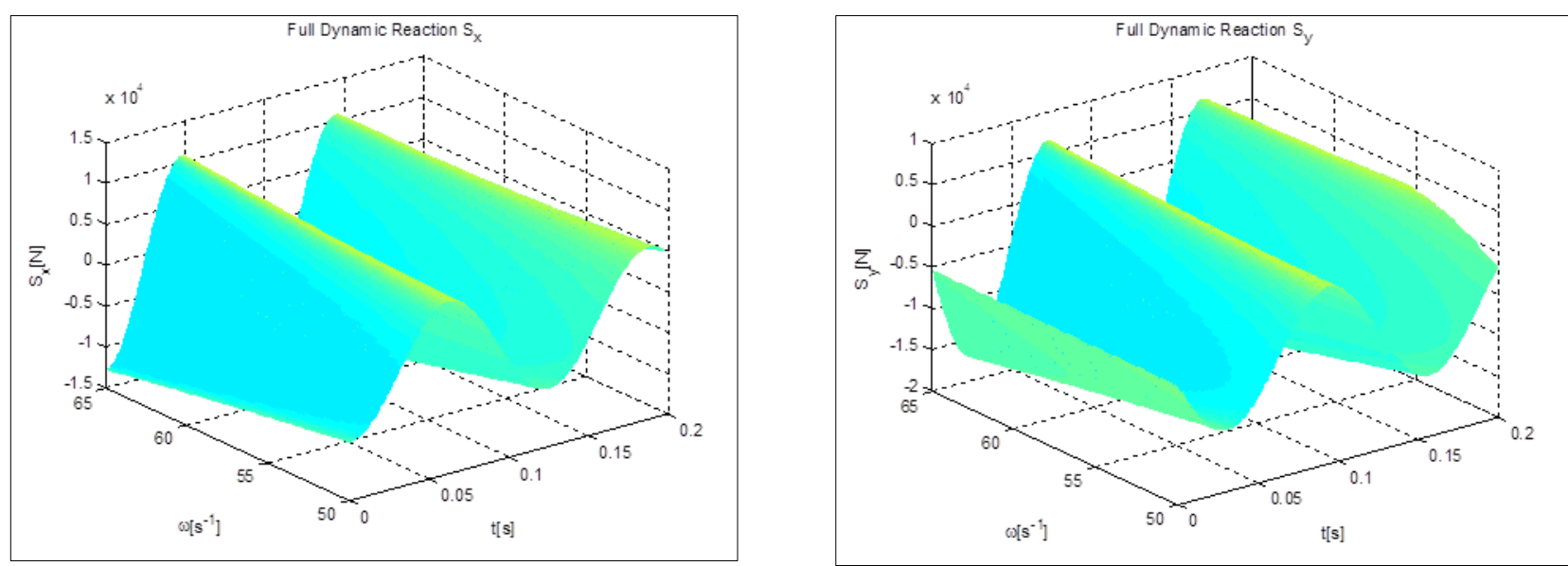

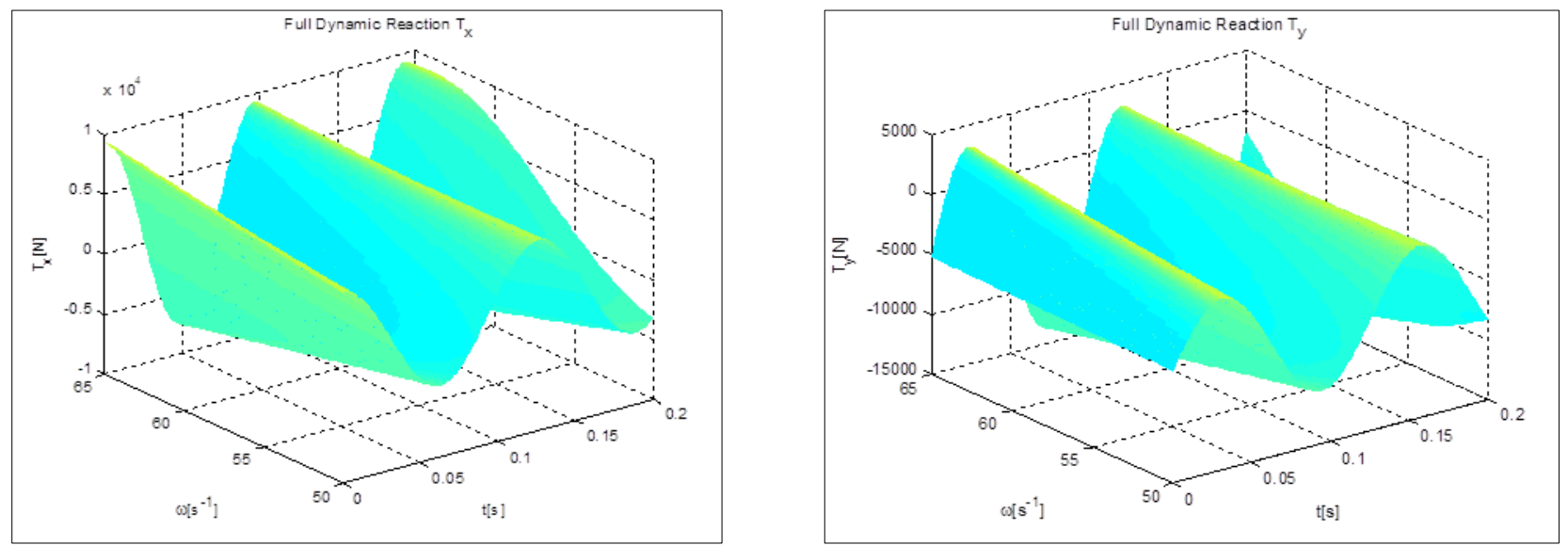

Figure 8 Spatial diagrams of the full dynamic reactions $S_{x}, S_{y}, T_{x}, T_{y}$

\section{Analysis of the obtained results}

In this article, plane and spatial diagrams are constructed using the obtained theoretical expressions for calculating the full dynamic reactions. These diagrams are shown in Figs. 7 and 8.

The plane diagrams presented in Fig.7 show the dependence of the dynamic reactions from the time $t$ on given values of the eccentricity $e$ and the angle $\alpha$. The diagrams, marked with 1 are drawn in a case where $e=0.001[m]$ and $\alpha=0.017[\mathrm{rad}]$. The diagrams, marked with 2 are drawn in a case where no eccentricity, i.e. $e=0$. The diagrams, marked with 3 are drawn in a case when the angle $\alpha$ is equal to zero, i.e. $\alpha=0$. The bearing load has a minimum value when $\alpha=0$. Obviously, the angle $\alpha$ has a greater influence on the size of the dynamic reactions than the eccentricity $e$. This fact can be used in the design of new band saw machines. These diagrams also show that dynamic reactions take on both positive and negative values. This is a very unfavorable operating mode because it can lead to the so-called material fatigue phenomenon and, consequently, to the destruction of the machine shaft.

The spatial diagrams presented in Fig. 8 show how the dynamic reactions change when the two arguments change, i.e., when the time $t$ and the angular velocity $\omega$ change. These diagrams are constructed when the linear and angular deviations assume maximum values. We can find that as the angular velocity increases, the magnitudes of the dynamic reactions also increase. This is due to the fact that in this case the magnitudes of inertial forces and moments increase.

The obtained theoretical expressions can be used in different cases.

- $\quad$ Strength dimensioning or strength check of the upper shaft of the band saw machine;

- Deformation check of the upper shaft of the band saw machine;

- Study of the transverse vibrations and spatial deformations of the upper shaft;

- Optimal placement of the leading wheel and suitable disposal of the bearing supports and achieving the minimum dimensions of the mechanical system.

\section{Symbols used in the article}

A Band saw blade

$B$ Width of the band saw blade $[m]$

$C_{4} x^{\prime} y^{\prime} z^{\prime}$ Coordinate system

$C_{4} \xi \eta \varsigma$ Coordinate system

$D$ Diameter of the leading wheel $[\mathrm{m}]$

$F_{4}^{e}$ Tensile force $[N]$ $a_{\rho} \quad$ Coefficient of the blunt teeth

$b \quad$ Width of the cutter $[m]$

$e \quad$ Eccentricity $[m]$

$g \quad$ Acceleration of gravity $\left[\mathrm{m} / \mathrm{s}^{2}\right]$

$k \quad$ Fictitious pressure $\left[N / m^{2}\right]$

$m_{4} \quad$ Mass of the leading wheel $[\mathrm{kg}]$ 
$G_{4}^{e}$ Weight of the leading wheel $[N]$

$H$ Thickness of the workpiece $[m]$

$J_{x z}^{(0)}, J_{y z}^{(0)}$ Centrifugal moments $\left[\mathrm{kgm}^{2}\right]$

$J_{x z}^{(0)}, J_{y z}^{(0)}$ Centrifugal moments $\left[\mathrm{kgm}^{2}\right]$

$J_{\xi}, J_{\zeta}$ Mass moments of inertia $\left[\mathrm{kgm}^{2}\right]$

$K_{\Delta(\lambda)}$ Specific work of the cutting $\left[\mathrm{J} / \mathrm{m}^{3}\right]$

$M_{R 4 x}^{e}, M_{R 4 y}^{e}, M_{R 4 z}^{e} \quad$ Moments $[\mathrm{Nm}]$

$M_{G 4 x}^{e}, M_{G 4 y}^{e}, M_{G 4 z}^{e} \quad$ Moments $[N m]$

$N_{c} \quad$ Cutting power $[k W]$

$\mathrm{O}_{4} x y z$ Fixed coordinate system

$O_{4} x_{1} y_{1} z_{1}$ Moving coordinate system

$P_{b}^{\tau} \quad$ Cutting force [N]

$R_{b}^{n} \quad$ Normal force [N]

$R_{\Sigma} \quad$ Total resistance force $[N]$

$S_{x}^{e}, S_{y}^{e}, S_{z}^{e}$

$T_{x}^{e}, T_{y}^{e}$

Dynamic reactions $[N]$

$S_{x}^{i}, S_{y}^{i}$

$T_{x}^{i}, T_{y}^{i}$

Dynamic reactions $[N]$

$S_{x}, S_{y}$

$T_{x}, T_{y}$

$V \quad$ Cutting speed $[\mathrm{m} / \mathrm{s}]$ $m \quad$ Coefficient

$p \quad$ Fictitious specific force $[\mathrm{N} / \mathrm{m}]$

$S$ Thickness of the band saw blades $[m]$

$t \quad$ Time $[s]$

$u \quad$ Feeding speed $[\mathrm{m} / \mathrm{s}]$

$u_{z \Delta}, u_{z \lambda}$ Feeding of one tooth $[m]$

$x, y, z \quad$ Coordinates axes

$x_{1}, y_{1}, z_{1} \quad$ Coordinates axes

$x^{\prime}, y^{\prime}, z^{\prime} \quad$ Coordinates axes

$x_{C_{4}}^{(0)}, y_{C_{4}}^{(0)}, z_{C_{4}}^{(0)}$ Coordinates of point $C_{4}[m]$

$\alpha \quad$ Angular deviation $[\mathrm{rad}]$

$\alpha_{\Delta(\lambda)}$ Friction intensity of the shavings [Pa]

$\xi, \zeta, \eta \quad$ Coordinates axes

$\varphi \quad$ Angle of rotation [ $\mathrm{rad}]$

$\varepsilon \quad$ Angular acceleration $\left[s^{-2}\right]$

$\omega \quad$ Angular velocity $\left[s^{-1}\right]$

\section{Conclusion}

In this paper, the dynamic processes that arise in the operating mode of big band saw machines are analyzed. Theoretical expressions for calculating the full dynamic reactions in the bearing supports are obtained. For this purpose, the dynamic reactions caused by external loads are analyzed and expressions for their calculation are obtained. The dynamic reactions caused by the kinematics and mass characteristics of the rotating disk are also analyzed. In this case, dependencies for calculating these reactions are obtained. They are used to determine the full dynamic reactions. The obtained theoretical expressions show that the full dynamic reactions change according to a harmonic law. The plane and spatial diagrams are drawn. The plane diagrams show that the angular deflections have a more significant influence on the magnitudes of the reactions than the linear deflections. Spatial diagrams show how the reactions are changed when the angular velocity change. We can see that the magnitudes of the reactions increase when the angular velocity increase. An analysis of the obtained results is made. Various applications of the theoretical expressions for calculating the full dynamic reactions are shown. The more important applications are strength check, deformation check, as well as investigation of the transverse vibrations and spatial deformations of the upper shaft. In conclusion, we can say that the results obtained in the article can be used in future research related to ensuring reliable and safe operation of big band saw machines.

\section{Compliance with ethical standards}

\section{Acknowledgments}


The author would like to express his acknowledgments to the Institute of Mechanics, Bulgarian Academy of Sciences, which is his workplace.

\section{Disclosure of conflict of interest}

The author of this article declares that he has no conflict of interest with anyone in conducting and completing this study.

\section{References}

[1] Zheng W, Ben-hao J, Xiao-jun Y. Dynamic tensile force survey of woodworking band saw blades. International Conference on Solid State and Materials Lecture, Notes in Information Technology. Nanjing, China. 2012; 22: 3137.

[2] Marchal R, Mothe F, Denaud L-E, Thibaut B, Bleron L. Cutting forces in wood machining - Basics and applications in industrial processes. A review COST Action E35 2004-2008: Wood machining - micromechanics and fracture. Holzforschung. 2009; 63(2): 157-167.

[3] Porankiewicz B, Goli G. Cutting forces by oak and douglas fir machining. Maderas. Ciencia y tecnología. 2014; 16(2): 199-216.

[4] Hlásková L, Orlowski K, Kopecký Z, Jedinák M. Sawing processes as a way of determining fracture toughness and shear yield stresses of wood. BioResources. 2015; 10(3): 5381-5394.

[5] Moradpour P, Scholz F, Doosthoseini K, Tarmian A. Measurement of wood cutting forces during bandsawing using piezoelectric dynamometer. Drvna Industrija. 2016; 67(1): 79-84.

[6] Krenke T, Frybort S, Müller U. Determining cutting force parameters by applying a system function. Journal Machining Science and Technology. 2017; 21(3): 436-451.

[7] Obreshkov P. Woodworking Machines. Sofia: Publishing House "BM". 1995.

[8] Marinov B. Dynamic and Shock Processes in Some Classes of Woodworking Machines. Analysis and Optimization. Germany: Omniscriptum Publishing Group /LAP LAMBERT Academic Publishing. 2018.

[9] Marinov B. Spatial deformations in the transmissions of certain classes of woodworking machines. Mechanism and Machine Theory. 2014; 82: 1-16.

[10] Pisarev A, Paraskov Ts, Bachvarov C. Course in Theoretical Mechanics. Second part-Dynamics. Sofia: State Publishing House Technics. 1988.

[11] Dreizler R, Lüdde C. Theoretical Mechanics: Theoretical Physics 1. Heidelberg: Springer. 2010.

[12] Porankiewicz B, Axelsson B, Grônlund A, Marklund B. Main and normal cutting forces by machining wood of pinus sylvestris. Bio Resources. 2011; 6(4): 3687-3713.

[13] Gochev Zh. Handbook for Exercise of Wood Cutting and Woodworking Tools. Sofia: Publishing House in LTU. 2005.

[14] Peters R. Band Saw Fundamentals: The Complete Guide. Hearst Communications Inc. 2006.

[15] Walker Turner. A Comprehensive Handbook on Uses and Applications of the Band Saw and Jig Saw. Literary Licensing LLC. 2013.

[16] Barcík Š. Experimental cutting on the table band-sawing machine. European Journal of Wood and Wood Products. 2003; 61(4): 313-320. 